\title{
Reliability of a series system with redundancy and one repair facility
}

\author{
Yuriy V. Zhernovyi ${ }^{\star}$ • Soltan A. Aliyev • Yaroslav I. \\ Yeleyko
}

Received: 13.03.2019 / Revised: 30.07.2020 / Accepted: 18.08.2020

\begin{abstract}
In this paper we propose a method for studying the reliability of series systems with redundancy and one repair facility. The approach based on the use of fictitious phases and hyperexponential approximations of arbitrary distributions by method of moments. We define conditions for the variation coefficients of the gamma distributions and Weibull distributions, for which the best accuracy of calculating the steady-state probabilities is achieved in comparison with the results of simulation modeling.
\end{abstract}

Keywords. reliability, recoverable system, redundancy, series system, hyperexponential approximation, fictitious phases, method of moments.

Mathematics Subject Classification (2010): 60G10, 60J28, 60K25, 93B40

\section{Introduction}

Series systems of identical independent units with a common group of redundant units are common in engineering practice. Formally, such a structure appears if a system consists of units of several types [4]. A set of units of the same type can be considered as a series system, for which there is a stock of spare units. It is reasonable to consider these spare units as unloaded; these units wait for being switched into operating position after one of operating units has failed. Failed units are directed to a repair shop, from where after recovery they again enter the systems stock. Switching of spare unit into an operating position is usually assumed instantaneous.

Recoverable series systems differ by their recovery processes. Assume that a system continues to stay in an operational state after failure, so during recovering a currently failed unit new failures may appear. In principle, in this case one can observe even a situation

\footnotetext{
* Corresponding author

Y.V. Zhernovyi

Ivan Franko National University of Lviv, Lviv, Ukraine

E-mail: yuriy.zhernovyy@lnu.edu.ua

S.A. Aliyev

Institute of Mathematics and Mechanics of NAS of Azerbaijan, AZ 1141, Baku, Azerbaijan

E-mail: soltanaliyev@yahoo.com

Y.I. Yeleyko

Ivan Franko National University of Lviv, Lviv, Ukraine

Jan Kochanowski University in Kielce, Kielce, Poland

E-mail: yikts@yahoo.com
} 
when all system units have failed. It can happen if, for instance, a recovery process is very slow. In addition, the number of repair facilities can be restricted, so failed units can form a queue for recovering. We restrict ourselves to one repair facility.

Consider a system that consists of $r=n+m$ identical units, namely, $n$ main operating units and $m$ unloaded redundant units. The system stops functioning in normal mode at the moment when the number of failed units reaches $m+1$. Assume that those $n-1$ units that were serviceable at the moment of system failure, in idle time during recovery, continue to operate and may fail.

If we consider the described system as a single-channel queueing system, then in the absence of redundant units $(m=0)$, it is a classical closed queueing system [2]. The closed system is also known as a system with a finite number of sources or the Engset system.

In most academic approaches, a random time to failure and a random recovery time are assumed exponentially distributed for all units that gives a possibility to use the Markov model for reliability study. In this paper we consider arbitrary distributions of the units' time to failure and recovery time.

The method of potentials was used in [5] to construct an algorithm that makes it possible to calculate the steady-state distribution of the number of failed units for a single-channel closed system with an exponential distribution of the units' time to failure and an arbitrary distribution of recovery time. This method is not suitable for arbitrary distributions of the units' time to failure.

In [2] we propose a method for calculating steady-state probability distributions of the closed queueing systems with exponential distribution of the time to failure and an arbitrary distribution of recovery time. The approach based on the use of fictitious phases and hyperexponential approximations by method of moments. We consider queueing systems with the number of channels $n=1,2$ and 3 .

Works $[1-3,6,7]$ show that the use of hyperexponential approximation $\left(H_{l}\right)$ makes it possible to determine with high accuracy the steady-state probabilities of non-Markovian queuing systems. These probabilities are calculated using solutions of a system of linear algebraic equations obtained by the method of fictitious phases. To find parameters of the $H_{l}$-approximation of a certain distribution it is sufficient to solve the system of equations of the moments method. For the values $V<1$ of the variation coefficient, roots of this system are complex-valued or paradoxical (i.e., negative or with probabilities that exceed the boundaries of the interval $[0,1]$ ), but in most cases as a result of summation of probabilities of microstates, their complex-valued and paradoxical parts are annihilated.

The purpose of the paper is to use of the hyperexponential approximation method for studying the reliability of series systems with redundancy and one repair facility with arbitrary distributions of the units' time to failure and recovery time. We define conditions for the variation coefficients of the gamma distributions and Weibull distributions, for which the best accuracy of calculating the steady-state probabilities is achieved in comparison with the results of simulation modeling.

Let $p_{k}$ be the steady-state probability of having $k$ failed units, then the stationary reliability indices of the system are determined by the formulas:

$$
\begin{gathered}
K=\sum_{k=0}^{m} p_{k}, \quad N=\sum_{k=0}^{r} k p_{k}, \quad Q=\sum_{k=1}^{r-1} k p_{k+1}, \quad W=Q / \Lambda, \quad \Lambda=\sum_{k=0}^{r-1} \Lambda_{k} p_{k}, \\
\Lambda_{k}= \begin{cases}n / T, & 0 \leq k \leq m ; \\
(r-k) / T, & m+1 \leq k \leq r-1 .\end{cases}
\end{gathered}
$$

Here $K$ is the stationary availability coefficient, $N$ is the average number of failed units, $Q$ is the average queue for recovering, $W$ is the mean waiting time for recovering and $T$ is the mean time to failure of an unit. 


\section{Equations for steady-state probabilities}

The hyperexponential distribution of order $l$ is a phase-type distribution and provides for choosing one of $l$ alternative phases by a random process. With probability $y_{i}$, the process is in the $i$ th phase during an exponentially distributed time with a parameter $\theta_{i}$.

Suppose that the units' time to failure is distributed according to the hyperexponential law $H_{4}$ with probabilities $\alpha_{i}$ and parameters $\lambda_{i}(1 \leq i \leq 4)$ and the units' recovery times are independent random variables distributed according to the hyperexponential law $H_{l}(l \geq 2)$ with probabilities $\beta_{s}$ and parameters $\mu_{s}(1 \leq s \leq l)$. Let us denote the corresponding queueing system by $H_{4} / H_{l} / 1 / r$ and we will use it for approximate calculation of the steady-state probabilities of the $G / G / 1 / r$ queueing system. We restrict ourselves to considering the $\mathrm{H}_{4}$ distribution, since calculations show that such an order of hyperexponential distribution provides sufficient accuracy for approximating an arbitrary distribution of the time to failure.

Let us enumerate the $H_{4} / H_{l} / 1 / r$ system's states as follows: $x_{0(i, j, u, v)}$ is the state, when there are 0 failed units, and $i, j, u, v$ are the numbers of units for which the time to failure is in the first, second, third and fourth phase, respectively; $x_{k(i, j, u, v, s)}$ is the state, when there are $k$ failed units $(1 \leq k \leq r)$, and $i, j, u, v$ are the numbers of units for which the time to failure is in the first, second, third and fourth phase, respectively, and $s$ is the phase number of recovery time. We denote by $p_{0(i, j, u, v)}$ and $p_{k(i, j, u, v, s)}$, steady-state probabilities that the system is in the each of these states respectively. Since the process of changing the states of the system is Markovian with continuous time, for the steady-state probabilities $p_{0(i, j, u, v)}$ and $p_{k(i, j, u, v, s)}$ we obtain a system of linear algebraic equations that follows from the Kolmogorov system of differential equations.

Let us write the equations corresponding to the states $x_{0(n, 0,0,0)}, x_{0(0, n, 0,0)}$, $x_{0(0,0, n, 0)}$ and $x_{0(0,0,0, n)}$ :

$$
\begin{array}{ll}
-n \lambda_{1} p_{0(n, 0,0,0)}+\sum_{i=1}^{l} \mu_{i} p_{1(n, 0,0,0, i)}=0, & -n \lambda_{2} p_{0(0, n, 0,0)}+\sum_{i=1}^{l} \mu_{i} p_{1(0, n, 0,0, i)}=0, \\
-n \lambda_{3} p_{0(0,0, n, 0)}+\sum_{i=1}^{l} \mu_{i} p_{1(0,0, n, 0, i)}=0, & -n \lambda_{4} p_{0(0,0,0, n)}+\sum_{i=1}^{l} \mu_{i} p_{1(0,0,0, n, i)}=0 .
\end{array}
$$

On the example of the equation corresponding to the state $x_{0(i, n-i, 0,0)}$, we show what is the structure of the equations for the states $x_{0(i, 0, n-i, 0)}, x_{0(i, 0,0, n-i)}$, $x_{0(0, i, n-i, 0)}, x_{0(0, i, 0, n-i)}$ and $x_{0(0,0, i, n-i)}$ :

$$
-\left(i \lambda_{1}+(n-i) \lambda_{2}\right) p_{0(i, n-i, 0,0)}+\sum_{j=1}^{l} \mu_{j} p_{1(i, n-i, 0,0, j)}=0, \quad 1 \leq i \leq n-1 .
$$

Let us write the equation corresponding to the state $x_{0(i, j, n-i-j, 0)}$ :

$$
\begin{array}{r}
-\left(i \lambda_{1}+j \lambda_{2}+(n-i-j) \lambda_{3}\right) p_{0(i, j, n-i-j, 0)}+\sum_{s=1}^{l} \mu_{s} p_{1(i, j, n-i-j, 0, s)}=0 \\
1 \leq i \leq n-2, \quad 1 \leq j \leq n-1-i .
\end{array}
$$

The equations for the states $x_{0(i, j, 0, n-i-j)}, x_{0(i, 0, j, n-i-j)}$ and $x_{0(0, i, j, n-i-j)}$ have a similar structure with (2.1). 
Let us write the equation corresponding to the state $x_{0(i, j, s, n-i-j-s)}$ :

$$
\begin{array}{r}
-\left(i \lambda_{1}+j \lambda_{2}+s \lambda_{3}+(n-i-j-s) \lambda_{4}\right) p_{0(i, j, s, n-i-j-s)}+\sum_{u=1}^{l} \mu_{u} p_{1(i, j, s, n-i-j-s, u)}=0 \\
1 \leq i \leq n-3, \quad 1 \leq j \leq n-2-i, \quad 1 \leq s \leq n-1-i-j .
\end{array}
$$

Let us give examples of equations corresponding to the states $x_{1(n, 0,0,0, i)}$, $x_{1(0, n, 0,0, i)}, \quad x_{1(0,0, n, 0, i)}, \quad x_{1(0,0,0, n, i)}, \quad x_{1(i, n-i, 0,0, j)}, \quad x_{1(i, 0, n-i, 0, j)}, \quad x_{1(i, 0,0, n-i, j)}$, $x_{1(0, i, n-i, 0, j)}, x_{1(0, i, 0, n-i, j)}, x_{1(0,0, i, n-i, j)}, x_{1(i, j, n-i-j, 0, s)}, x_{1(i, j, 0, n-i-j, s)}, x_{1(i, 0, j, n-i-j, s)}$, $x_{1(0, i, j, n-i-j, s)}$ and $x_{1(i, j, s, n-i-j-s, v)}$ :

$$
\begin{aligned}
& -\left(n \lambda_{1}+\mu_{i}\right) p_{1(n, 0,0,0, i)}+\beta_{i} \sum_{j=1}^{l} \mu_{j} p_{2(n, 0,0,0, j)}+\alpha_{1} \beta_{i}\left(n \lambda_{1} p_{0(n, 0,0,0)}\right. \\
& \left.+\lambda_{2} p_{0(n-1,1,0,0)}+\lambda_{3} p_{0(n-1,0,1,0)}+\lambda_{4} p_{0(n-1,0,0,1)}\right)=0, \quad 1 \leq i \leq l \\
& -\left(i \lambda_{1}+(n-i) \lambda_{2}+\mu_{j}\right) p_{1(i, n-i, 0,0, j)}+\beta_{j} \sum_{s=1}^{l} \mu_{s} p_{2(i, n-i, 0,0, s)}+\alpha_{1} \beta_{j}\left(i \lambda_{1} p_{0(i, n-i, 0,0)}\right. \\
& \left.+(n+1-i) \lambda_{2} p_{0(i-1, n+1-i, 0,0)}+\lambda_{3} p_{0(i-1, n-i, 1,0)}+\lambda_{4} p_{0(i-1, n-i, 0,1)}\right) \\
& +\alpha_{2} \beta_{j}\left((i+1) \lambda_{1} p_{0(i+1, n-1-i, 0,0)}\right. \\
& \left.+(n-i) \lambda_{2} p_{0(i, n-i, 0,0)}+\lambda_{3} p_{0(i, n-1-i, 1,0)}+\lambda_{4} p_{0(i, n-1-i, 0,1)}\right)=0
\end{aligned}
$$$$
1 \leq i \leq n-1, \quad 1 \leq j \leq l \text {; }
$$$$
-\left(i \lambda_{1}+j \lambda_{2}+(n-i-j) \lambda_{3}+\mu_{s}\right) p_{1(i, j, n-i-j, 0, s)}+\beta_{s} \sum_{u=1}^{l} \mu_{u} p_{2(i, j, n-i-j, 0, u)}
$$$$
+\alpha_{1} \beta_{s}\left(i \lambda_{1} p_{0(i, j, n-i-j, 0)}+(j+1) \lambda_{2} p_{0(i-1, j+1, n-i-j, 0)}\right.
$$$$
\left.+(n+1-i-j) \lambda_{3} p_{0(i-1, j, n+1-i-j, 0)}+\lambda_{4} p_{0(i-1, j, n-i-j, 1)}\right)
$$$$
+\alpha_{2} \beta_{s}\left((i+1) \lambda_{1} p_{0(i+1, j-1, n-i-j, 0)}+j \lambda_{2} p_{0(i, j, n-i-j, 0)}\right.
$$$$
\left.+(n+1-i-j) \lambda_{3} p_{0(i, j-1, n+1-i-j, 0)}+\lambda_{4} p_{0(i, j-1, n-i-j, 1)}\right)
$$$$
+\alpha_{3} \beta_{s}\left((i+1) \lambda_{1} p_{0(i+1, j, n-1-i-j, 0)}+(j+1) \lambda_{2} p_{0(i, j+1, n-1-i-j, 0)}\right.
$$$$
\left.+(n-i-j) \lambda_{3} p_{0(i, j, n-i-j, 0)}+\lambda_{4} p_{0(i, j, n-1-i-j, 1)}\right)=0,
$$

$1 \leq i \leq n-2, \quad 1 \leq j \leq n-1-i, \quad 1 \leq s \leq l$;

$-\left(i \lambda_{1}+j \lambda_{2}+s \lambda_{3}+(n-i-j-s) \lambda_{4}+\mu_{u}\right) p_{1(i, j, s, n-i-j-s, u)}$

$$
+\beta_{u} \sum_{v=1}^{l} \mu_{v} p_{2(i, j, s, n-i-j-s, v)}
$$

$+\beta_{u} \alpha_{1}\left(i \lambda_{1} p_{0(i, j, s, n-i-j-s)}+(j+1) \lambda_{2} p_{0(i-1, j+1, s, n-i-j-s)}\right.$

$\left.+(s+1) \lambda_{3} p_{0(i-1, j, s+1, n-i-j-s)}(n+1-i-j-s) \lambda_{4} p_{0(i-1, j, s, n+1-i-j-s)}\right)$

$+\beta_{u} \alpha_{2}\left((i+1) \lambda_{1} p_{0(i+1, j-1, s, n-i-j-s)}+j \lambda_{2} p_{0(i, j, s, n-i-j-s)}\right.$

$\left.+(s+1) \lambda_{3} p_{0(i, j-1, s+1, n-i-j-s)}+(n+1-i-j-s) \lambda_{4} p_{0(i, j-1, s, n+1-i-j-s)}\right)$

$+\beta_{u} \alpha_{3}\left((i+1) \lambda_{1} p_{0(i+1, j, s-1, n-i-j-s)}+(j+1) \lambda_{2} p_{0(i, j+1, s-1, n-i-j-s)}\right.$ 


$$
\begin{aligned}
& \left.+s \lambda_{3} p_{0(i, j, s, n-i-j-s)}+(n+1-i-j-s) \lambda_{4} p_{0(i, j, s-1, n+1-i-j-s)}\right) \\
& +\beta_{u} \alpha_{4}\left((i+1) \lambda_{1} p_{0(i+1, j, s, n-1-i-j-s)}+(j+1) \lambda_{2} p_{0(i, j+1, s, n-1-i-j-s)}\right. \\
& \left.+(s+1) \lambda_{3} p_{0(i, j, s+1, n-1-i-j-s)}+(n-i-j-s) \lambda_{4} p_{0(i, j, s, n-i-j-s)}\right)=0, \\
& 1 \leq i \leq n-3, \quad 1 \leq j \leq n-2-i, \quad 1 \leq s \leq n-1-i-j, \quad 1 \leq u \leq l .
\end{aligned}
$$

The structure of equations corresponding to the states $x_{k(n, 0,0,0, i)}, x_{k(0, n, 0,0, i)}$, $x_{k(0,0, n, 0, i)}, \quad x_{k(0,0,0, n, i)}, \quad x_{k(i, n-i, 0,0, j)}, \quad x_{k(i, 0, n-i, 0, j)}, \quad x_{k(i, 0,0, n-i, j)}, \quad x_{k(0, i, n-i, 0, j)}$, $x_{k(0, i, 0, n-i, j)}, \quad x_{k(0,0, i, n-i, j)}, \quad x_{k(i, j, n-i-j, 0, s)}, \quad x_{k(i, j, 0, n-i-j, s)}, \quad x_{k(i, 0, j, n-i-j, s)}$, $x_{k(0, i, j, n-i-j, s)}$ and $x_{k(i, j, s, n-i-j-s, v)}$ for $k \in\{2,3, \ldots, m-1\}$ differs from the structure of equations (2.2) only in the absence of the multiplier $\beta_{i}$ before the probabilities $p_{k-1(., ., ., ., i)}$ :

$$
-\left(n \lambda_{1}+\mu_{i}\right) p_{k(n, 0,0,0, i)}+\beta_{i} \sum_{j=1}^{l} \mu_{j} p_{k+1(n, 0,0,0, j)}+\alpha_{1}\left(n \lambda_{1} p_{k-1(n, 0,0,0, i)}\right.
$$

$\left.+\lambda_{2} p_{k-1(n-1,1,0,0, i)}+\lambda_{3} p_{k-1(n-1,0,1,0, i)}+\lambda_{4} p_{k-1(n-1,0,0,1, i)}\right)=0$,

$2 \leq k \leq m-1, \quad 1 \leq i \leq l$;

$$
\begin{aligned}
& -\left(i \lambda_{1}+(n-i) \lambda_{2}+\mu_{j}\right) p_{k(i, n-i, 0,0, j)}+\beta_{j} \sum_{s=1}^{l} \mu_{s} p_{k+1(i, n-i, 0,0, s)} \\
& +\alpha_{1}\left(i \lambda_{1} p_{k-1(i, n-i, 0,0, j)}+(n+1-i) \lambda_{2} p_{k-1(i-1, n+1-i, 0,0, j)}+\lambda_{3} p_{k-1(i-1, n-i, 1,0, j)}\right. \\
& \left.+\lambda_{4} p_{k-1(i-1, n-i, 0,1, j)}\right) \\
& +\alpha_{2}\left((i+1) \lambda_{1} p_{k-1(i+1, n-1-i, 0,0, j)}+(n-i) \lambda_{2} p_{k-1(i, n-i, 0,0, j)}+\lambda_{3} p_{k-1(i, n-1-i, 1,0, j)}\right. \\
& \left.+\lambda_{4} p_{k-1(i, n-1-i, 0,1, j)}\right)=0, \quad 2 \leq k \leq m-1, \quad 1 \leq i \leq n-1, \quad 1 \leq j \leq l ; \quad \ldots .
\end{aligned}
$$

Let us give examples of equations corresponding to the states $x_{k(r-k, 0,0,0, i)}$, $x_{k(0, r-k, 0,0, i)}, \quad x_{k(0,0, r-k, 0, i)}, \quad x_{k(0,0,0, r-k, i)}, \quad x_{k(i, r-k-i, 0,0, j)}, \quad x_{k(i, 0, r-k-i, 0, j)}$, $x_{k(i, 0,0, r-k-i, j)}, \quad x_{k(0, i, r-k-i, 0, j)}, \quad x_{k(0, i, 0, r-k-i, j)}, \quad x_{k(0,0, i, r-k-i, j)}, \quad x_{k(i, j, r-k-i-j, 0, s)}$, $x_{k(i, j, 0, r-k-i-j, s)}, \quad x_{k(i, 0, j, r-k-i-j, s)}, \quad x_{k(0, i, j, r-k-i-j, s)}$ and $x_{k(i, j, s, r-k-i-j-s, v)}$ for $k \in\{m+1, m+2, \ldots, r-1\}$

$$
\begin{aligned}
& -\left((r-k) \lambda_{1}+\mu_{i}\right) p_{k(r-k, 0,0,0, i)}+\alpha_{1} \beta_{i} \sum_{j=1}^{l} \mu_{j} p_{k+1(r-k-1,0,0,0, j)} \\
& +(r-k+1) \lambda_{1} p_{k-1(r-k+1,0,0,0, i)}+\lambda_{2} p_{k-1(r-k, 1,0,0, i)}+\lambda_{3} p_{k-1(r-k, 0,1,0, i)} \\
& +\lambda_{4} p_{k-1(r-k, 0,0,1, i)}=0, \quad m+1 \leq k \leq r-1, \quad 1 \leq i \leq l ; \\
& -\left(i \lambda_{1}+(r-k-i) \lambda_{2}+\mu_{j}\right) p_{k(i, r-k-i, 0,0, j)}+\beta_{j} \sum_{s=1}^{l} \mu_{s}\left(\alpha_{1} p_{k+1(i-1, r-k-i, 0,0, s)}\right. \\
& \left.+\alpha_{2} p_{k+1(i, r-k-i-1,0,0, s)}\right)+(i+1) \lambda_{1} p_{k-1(i+1, r-k-i, 0,0, j)} \\
& +(r-k-i+1) \lambda_{2} p_{k-1(i, r-k-i+1,0,0, j)}+\lambda_{3} p_{k-1(i, r-k-i, 1,0, j)} \\
& +\lambda_{4} p_{k-1(i, r-k-i, 0,1, j)}=0, \quad m+1 \leq k \leq r-2, \quad 1 \leq i \leq r-k-1, \quad 1 \leq j \leq l ; \\
& -\left(i \lambda_{1}+j \lambda_{2}+(r-k-i-j) \lambda_{3}+\mu_{s}\right) p_{k(i, j, r-k-i-j, 0, s)} \\
& +\beta_{s} \sum_{u=1}^{l} \mu_{u}\left(\alpha_{1} p_{k+1(i-1, j, r-k-i-j, 0, u)}+\alpha_{2} p_{k+1(i, j-1, r-k-i-j, 0, u)}\right. \\
& \left.+\alpha_{3} p_{k+1(i, j, r-k-i-j-1,0, u)}\right)+(i+1) \lambda_{1} p_{k-1(i+1, j, r-k-i-j, 0, s)}
\end{aligned}
$$




$$
\begin{aligned}
& +(j+1) \lambda_{2} p_{k-1(i, j+1, r-k-i-j, 0, s)}+(r-k-i-j+1) \lambda_{3} p_{k-1(i, j, r-k-i-j+1,0, s)} \\
& +\lambda_{4} p_{k-1(i, j, r-k-i-j, 1, s)}=0 \\
& m+1 \leq k \leq r-3, \quad 1 \leq i \leq r-k-2, \quad 1 \leq j \leq r-k-i-1, \quad 1 \leq s \leq l \\
& -\left(i \lambda_{1}+j \lambda_{2}+s \lambda_{3}+(r-k-i-j-s) \lambda_{4}+\mu_{u}\right) p_{k(i, j, s, r-k-i-j-s, u)} \\
& +\beta_{u} \sum_{v=1}^{l} \mu_{v}\left(\alpha_{1} p_{k+1(i-1, j, s, r-k-i-j-s, v)}+\alpha_{2} p_{k+1(i, j-1, s, r-k-i-j-s, v)}\right. \\
& \left.+\alpha_{3} p_{k+1(i, j, s-1, r-k-i-j-s, v)}+\alpha_{4} p_{k+1(i, j, s, r-k-i-j-s-1, v)}\right) \\
& +(i+1) \lambda_{1} p_{k-1(i+1, j, s, r-k-i-j-s, u)}+(j+1) \lambda_{2} p_{k-1(i, j+1, s, r-k-i-j-s, u)} \\
& +(s+1) \lambda_{3} p_{k-1(i, j, s+1, r-k-i-j-s, u)} \\
& +(r-k-i-j-s+1) \lambda_{4} p_{k-1(i, j, s, r-k-i-j-s+1, u)}=0 \\
& m+1 \leq k \leq r-4, \quad 1 \leq i \leq r-k-3, \quad 1 \leq j \leq r-k-i-2 \\
& \quad 1 \leq s \leq r-k-i-j-1, \quad 1 \leq u \leq l .
\end{aligned}
$$

Let us write the equation corresponding to the state $x_{r(0,0,0,0, i)}$ :

$$
\begin{aligned}
-\mu_{i} p_{r(0,0,0,0, i)}+\lambda_{1} p_{r-1(1,0,0,0, i)} & \lambda_{2} p_{r-1(0,1,0,0, i)}+\lambda_{3} p_{r-1(0,0,1,0, i)} \\
& +\lambda_{4} p_{r-1(0,0,0,1, i)}=0, \quad 1 \leq i \leq l .
\end{aligned}
$$

The normalization condition closes the system of equations for steady-state probabilities:

$$
\begin{array}{r}
\sum_{i=0}^{n} \sum_{j=0}^{n-i} \sum_{s=0}^{n-i-j} p_{0(i, j, s, n-i-j-s)}+\sum_{k=1}^{m} \sum_{i=0}^{n} \sum_{j=0}^{n-i} \sum_{s=0}^{n-i-j} \sum_{u=1}^{l} p_{k(i, j, s, n-i-j-s, u)} \\
+\sum_{k=m+1}^{r} \sum_{i=0}^{r-k} \sum_{j=0}^{r-k-i r-k-i-j} \sum_{s=0}^{l} \sum_{u=1}^{l} p_{k(i, j, s, r-k-i-j-s, u)}=1 .
\end{array}
$$

Solving the system obtained by the method of fictitious phases, we find the steady-state probabilities $p_{k}$ of having $k$ failed units, using the formulas

$$
\begin{array}{r}
p_{0}=\sum_{i=0}^{n} \sum_{j=0}^{n-i} \sum_{s=0}^{n-i-j} p_{0(i, j, s, n-i-j-s)}, \\
p_{k}=\sum_{i=0}^{n} \sum_{j=0}^{n-i} \sum_{s=0}^{n-i-j} \sum_{u=1}^{l} p_{k(i, j, s, n-i-j-s, u)}, \quad 1 \leq k \leq m ; \\
p_{k}=\sum_{i=0}^{r-k} \sum_{j=0}^{r-k-i} \sum_{s=0}^{r-k-i-j} \sum_{u=1}^{l} p_{k(i, j, s, r-k-i-j-s, u)}, \quad m+1 \leq k \leq r .
\end{array}
$$

\section{Numerical results}

Let $\Gamma(V), W(V)$ and $U[a, b]$ denote the gamma distribution, Weibull distribution with coefficients of variation $V$, and uniform distribution on the interval $[a, b]$, respectively. 
Table 1 List of distributions $D_{1}$ and $D_{2}$ for which condition $\Delta_{(6,5)}<10^{-4}$ holds

\begin{tabular}{llllll}
\hline$D_{1}$ & $D_{2}$ & Values of $V_{2}$ & $D_{1}$ & $D_{2}$ & Values of $V_{1}$ \\
\hline$\Gamma(0.001)$ & $\Gamma\left(V_{2}\right)$ & {$[0.7,1.5]$} & $\Gamma\left(V_{1}\right)$ & $\Gamma(0.001)$ & $0.4,0.51,[0.7,100]$ \\
$\Gamma(0.001)$ & $W\left(V_{2}\right)$ & {$[0.8,1.2]$} & $W\left(V_{1}\right)$ & $\Gamma(0.001)$ & {$[0.4,10]$} \\
$\Gamma(0.4)$ & $\Gamma\left(V_{2}\right)$ & {$[0.001,0.3],[0.51,1.6]$} & $\Gamma\left(V_{1}\right)$ & $\Gamma(0.4)$ & $0.3,[0.8,100]$ \\
$\Gamma(0.4)$ & $W\left(V_{2}\right)$ & {$[0.001,1.3]$} & $W\left(V_{1}\right)$ & $\Gamma(0.4)$ & {$[0.3,10]$} \\
$W(0.4)$ & $\Gamma\left(V_{2}\right)$ & {$[0.001,1.6]$} & $W\left(V_{1}\right)$ & $W(0.4)$ & {$[0.3,10]$} \\
$W(0.4)$ & $W\left(V_{2}\right)$ & {$[0.001,1.3]$} & $\Gamma\left(V_{1}\right)$ & $W(0.4)$ & {$[0.3,100]$} \\
$U[0,8]$ & $\Gamma\left(V_{2}\right)$ & {$[0.001,1.4]$} & $\Gamma\left(V_{1}\right)$ & $U[0,2]$ & {$[0.3,100]$} \\
$U[0,8]$ & $W\left(V_{2}\right)$ & {$[0.001,1.3]$} & $W\left(V_{1}\right)$ & $U[0,2]$ & {$[0.3,10]$} \\
$\Gamma(0.8)$ & $\Gamma\left(V_{2}\right)$ & {$[0.001,1.8]$} & $\Gamma\left(V_{1}\right)$ & $\Gamma(0.8)$ & {$[0.001,100]$} \\
$\Gamma(0.8)$ & $W\left(V_{2}\right)$ & {$[0.001,1.4]$} & $W\left(V_{1}\right)$ & $\Gamma(0.8)$ & {$[0.001,10]$} \\
$W(0.8)$ & $\Gamma\left(V_{2}\right)$ & {$[0.001,1.8]$} & $\Gamma\left(V_{1}\right)$ & $W(0.8)$ & {$[0.001,100]$} \\
$W(0.8)$ & $W\left(V_{2}\right)$ & {$[0.001,1.4]$} & $W\left(V_{1}\right)$ & $W(0.8)$ & {$[0.001,10]$} \\
$\Gamma(1.3)$ & $\Gamma\left(V_{2}\right)$ & {$[0.001,1.6]$} & $\Gamma\left(V_{1}\right)$ & $\Gamma(1.3)$ & {$[0.001,100]$} \\
$\Gamma(1.3)$ & $W\left(V_{2}\right)$ & {$[0.001,1.4]$} & $W\left(V_{1}\right)$ & $\Gamma(1.3)$ & {$[0.001,10]$} \\
$W(1.3)$ & $\Gamma\left(V_{2}\right)$ & {$[0.001,1.7]$} & $\Gamma\left(V_{1}\right)$ & $W(1.3)$ & {$[0.4,100]$} \\
$W(1.3)$ & $W\left(V_{2}\right)$ & {$[0.001,1.4]$} & $W\left(V_{1}\right)$ & $W(1.3)$ & {$[0.4,10]$} \\
$\Gamma(100)$ & $\Gamma\left(V_{2}\right)$ & {$[0.001,1.5]$} & $\Gamma\left(V_{1}\right)$ & $\Gamma(2)$ & - \\
$\Gamma(100)$ & $W\left(V_{2}\right)$ & {$[0.001,1.3]$} & $W\left(V_{1}\right)$ & $\Gamma(2)$ & - \\
$W(10)$ & $\Gamma\left(V_{2}\right)$ & {$[0.001,1.8]$} & $\Gamma\left(V_{1}\right)$ & $W(2)$ & - \\
$W(10)$ & $W\left(V_{2}\right)$ & {$[0.001,1.4]$} & $W\left(V_{1}\right)$ & $W(2)$ & - \\
\hline
\end{tabular}

In this section we present the results of using the $H_{4} / H_{5} / 1 / r$ and $H_{4} / H_{6} / 1 / r$ systems to calculate the probabilities $p_{k}$ for the $G / G / 1 / r$ series recoverable system with redundancy. We consider the gamma distributions, Weibull distributions and uniform distributions of units' time to failure (named distribution $D_{1}$ ) and units' recovery time (named distribution $D_{2}$ ).

For the $M / G / 1 / r$ queueing systems the deviation $\Delta_{\text {sim }}=\sum_{k=0}^{r}\left|p_{k}-p_{k(s i m)}\right|$ of distribution $\left\{p_{k(s i m)}\right\}$, obtained using the GPSS World simulation system [8], from the distribution $\left\{p_{k}\right\}$, obtained using the method of potentials, exceeds $10^{-4}$ [7]. This statement is confirmed if we calculate $\Delta_{\text {sim }}$ for the considered series system with exponential distributions of the units' time to failure and recovery time. The GPSS World simulation model of the series recoverable system with redundancy is constructed in [9].

We determine the values of the variation coefficients of the $\Gamma(V)$ and $W(V)$ distributions, for which the condition $\Delta_{(6,5)}<10^{-4}$ holds. If this condition is fulfilled, the distribution $\left\{p_{k(6)}\right\}$ is a more accurate approximation to the true steady-state distribution $\left\{p_{k}\right\}$, than the distribution obtained using the GPSS World simulation system. Here $\Delta_{(6,5)}=\sum_{k=0}^{r}\left|p_{k(6)}-p_{k(5)}\right|$ gives an opportunity to estimate the deviation of distributions $\left\{p_{k(6)}\right\}$ from distributions $\left\{p_{k(5)}\right\}$, where $p_{k(l)}$ are values of probabilities $p_{k}$ obtained using the $H_{4} / H_{l} / 1 / r$ system as an approximation of the $G / G / 1 / r$ system.

Let us take $n=4, m=3, T=4$ and $\tau=1$, where $\tau$ is the mean units' recovery time. Thus, the average values of the distributions $D_{1}$ and $D_{2}$ are equal to 4 and 1 respectively.

The numerical results are presented in Table 1 . Of all the intervals corresponding to the $\Gamma(V)$ distributions, the values $V=0.5$ and $V=1 / \sqrt{2}$ of the variation coefficient should be excluded, since for the $\Gamma(0.5)$ distribution it is not possible to construct approximations with the help of hyperexponential distributions of order higher than the third, and hyperexponential approximations do not exist for the $\Gamma(1 / \sqrt{2})$ distribution.

The data in Table 1 show that a high accuracy of approximation is achieved for a wider range of values of the variation coefficient of the distribution $D_{1}$ compared to the distribution $D_{2}$. Namely, for the distribution $D_{1}$, the maximum interval for the variation coefficient 
Table 2 Results of the calculation of steady-state reliability indices for different distributions $D_{1}$ and $D_{2}$

\begin{tabular}{|c|c|c|c|c|c|}
\hline$D_{1}$ & $D_{2}$ & $N$ & $K$ & $W$ & $\Delta_{(6,5)}$ \\
\hline$\Gamma(0.001)$ & $W(0.6)$ & 2.419 & 0.798 & 1.583 & $2.30 \cdot 10^{-6}$ \\
\hline$\Gamma(0.001)$ & $\Gamma(0.7)$ & 2.436 & 0.776 & 1.641 & $3.56 \cdot 10^{-8}$ \\
\hline$\Gamma(0.001)$ & $W(0.8)$ & 2.484 & 0.748 & 1.737 & $2.70 \cdot 10^{-6}$ \\
\hline$\Gamma(0.001)$ & $\Gamma(1.3)$ & 2.654 & 0.670 & 2.169 & $3.67 \cdot 10^{-5}$ \\
\hline$\Gamma(0.001)$ & $\Gamma(1.5)$ & 2.720 & 0.649 & 2.347 & $8.90 \cdot 10^{-5}$ \\
\hline$W(0.4)$ & $\Gamma(0.001)$ & 2.305 & 0.868 & 1.396 & $2.41 \cdot 10^{-6}$ \\
\hline$W(0.5)$ & $\Gamma(0.001)$ & 2.347 & 0.834 & 1.472 & $3.54 \cdot 10^{-7}$ \\
\hline$\Gamma(0.7)$ & $\Gamma(0.001)$ & 2.425 & 0.778 & 1.617 & $2.86 \cdot 10^{-6}$ \\
\hline$\Gamma(0.8)$ & $\Gamma(0.001)$ & 2.461 & 0.753 & 1.690 & $4.69 \cdot 10^{-7}$ \\
\hline$\Gamma(1.5)$ & $\Gamma(0.001)$ & 2.696 & 0.628 & 2.177 & $9.18 \cdot 10^{-6}$ \\
\hline$W(10)$ & $\Gamma(0.001)$ & 2.659 & 0.645 & 2.100 & $3.88 \cdot 10^{-8}$ \\
\hline$\Gamma(100)$ & $\Gamma(0.001)$ & 3.284 & 0.363 & 3.599 & $9.28 \cdot 10^{-6}$ \\
\hline$\Gamma(0.4)$ & $\Gamma(0.1)$ & 2.321 & 0.864 & 1.415 & $2.26 \cdot 10^{-6}$ \\
\hline$\Gamma(0.4)$ & $\Gamma(0.3)$ & 2.356 & 0.834 & 1.480 & $6.06 \cdot 10^{-7}$ \\
\hline$\Gamma(0.4)$ & $W(0.4)$ & 2.391 & 0.811 & 1.542 & $8.54 \cdot 10^{-7}$ \\
\hline$\Gamma(0.4)$ & $W(1.3)$ & 2.652 & 0.667 & 2.177 & $7.70 \cdot 10^{-5}$ \\
\hline$\Gamma(0.4)$ & $\Gamma(1.6)$ & 2.755 & 0.634 & 2.450 & $7.10 \cdot 10^{-5}$ \\
\hline$\Gamma(0.3)$ & $\Gamma(0.4)$ & 2.359 & 0.833 & 1.484 & $2.30 \cdot 10^{-5}$ \\
\hline$W(0.3)$ & $\Gamma(0.4)$ & 2.356 & 0.833 & 1.482 & $2.13 \cdot 10^{-6}$ \\
\hline$\Gamma(0.8)$ & $\Gamma(0.4)$ & 2.498 & 0.731 & 1.770 & $4.40 \cdot 10^{-7}$ \\
\hline$W(10)$ & $\Gamma(0.4)$ & 2.672 & 0.640 & 2.140 & $8.02 \cdot 10^{-7}$ \\
\hline$\Gamma(100)$ & $\Gamma(0.4)$ & 3.281 & 0.364 & 3.595 & $4.06 \cdot 10^{-5}$ \\
\hline$W(0.4)$ & $W(0.1)$ & 2.312 & 0.864 & 1.407 & $2.12 \cdot 10^{-6}$ \\
\hline$W(0.4)$ & $\Gamma(0.49)$ & 2.408 & 0.792 & 1.586 & $4.02 \cdot 10^{-5}$ \\
\hline$W(0.4)$ & $W(1.3)$ & 2.655 & 0.665 & 2.181 & $7.12 \cdot 10^{-5}$ \\
\hline$W(0.4)$ & $\Gamma(1.6)$ & 2.757 & 0.632 & 2.454 & $6.88 \cdot 10^{-5}$ \\
\hline$\Gamma(0.3)$ & $W(0.4)$ & 2.371 & 0.831 & 1.497 & $3.18 \cdot 10^{-6}$ \\
\hline$W(0.3)$ & $W(0.4)$ & 2.368 & 0.831 & 1.495 & $2.85 \cdot 10^{-6}$ \\
\hline$\Gamma(0.6)$ & $W(0.4)$ & 2.442 & 0.769 & 1.650 & $3.28 \cdot 10^{-6}$ \\
\hline$W(10)$ & $W(0.4)$ & 2.674 & 0.639 & 2.142 & $3.69 \cdot 10^{-8}$ \\
\hline$\Gamma(100)$ & $W(0.4)$ & 3.281 & 0.364 & 3.595 & $3.11 \cdot 10^{-6}$ \\
\hline$U[0,8]$ & $W(0.1)$ & 2.376 & 0.801 & 1.542 & $1.08 \cdot 10^{-6}$ \\
\hline$U[0,8]$ & $U[0,2]$ & 2.511 & 0.731 & 1.782 & $6.37 \cdot 10^{-6}$ \\
\hline$U[0,8]$ & $W(0.9)$ & 2.588 & 0.687 & 1.978 & $1.64 \cdot 10^{-7}$ \\
\hline$U[0,8]$ & $\Gamma(1.4)$ & 2.729 & 0.633 & 2.350 & $1.19 \cdot 10^{-5}$ \\
\hline$\Gamma(0.3)$ & $U[0,2]$ & 2.463 & 0.775 & 1.655 & $2.83 \cdot 10^{-5}$ \\
\hline$W(0.4)$ & $U[0,2]$ & 2.474 & 0.763 & 1.688 & $7.91 \cdot 10^{-6}$ \\
\hline$W(10)$ & $U[0,2]$ & 2.697 & 0.631 & 2.199 & $5.50 \cdot 10^{-7}$ \\
\hline$\Gamma(100)$ & $U[0,2]$ & 3.273 & 0.366 & 3.584 & $2.97 \cdot 10^{-5}$ \\
\hline$\Gamma(0.8)$ & $W(1.3)$ & 2.702 & 0.640 & 2.287 & $3.23 \cdot 10^{-5}$ \\
\hline$\Gamma(0.8)$ & $\Gamma(1.4)$ & 2.740 & 0.628 & 2.381 & $9.93 \cdot 10^{-6}$ \\
\hline$\Gamma(0.8)$ & $W(1.4)$ & 2.722 & 0.634 & 2.347 & $9.67 \cdot 10^{-5}$ \\
\hline$\Gamma(0.8)$ & $\Gamma(1.8)$ & 2.834 & 0.602 & 2.666 & $7.99 \cdot 10^{-5}$ \\
\hline$\Gamma(0.6)$ & $\Gamma(0.8)$ & 2.543 & 0.709 & 1.880 & $1.11 \cdot 10^{-6}$ \\
\hline$\Gamma(1.5)$ & $\Gamma(0.8)$ & 2.757 & 0.603 & 2.344 & $2.27 \cdot 10^{-7}$ \\
\hline$W(10)$ & $\Gamma(0.8)$ & 2.714 & 0.625 & 2.259 & $3.72 \cdot 10^{-7}$ \\
\hline$\Gamma(100)$ & $\Gamma(0.8)$ & 3.271 & 0.367 & 3.582 & $3.80 \cdot 10^{-7}$ \\
\hline$W(0.8)$ & $W(1.4)$ & 2.726 & 0.632 & 2.355 & $9.58 \cdot 10^{-5}$ \\
\hline$W(0.8)$ & $\Gamma(1.8)$ & 2.837 & 0.600 & 2.671 & $7.56 \cdot 10^{-5}$ \\
\hline$\Gamma(0.6)$ & $W(0.8)$ & 2.549 & 0.706 & 1.888 & $3.59 \cdot 10^{-6}$ \\
\hline$W(10)$ & $W(0.8)$ & 2.716 & 0.624 & 2.263 & $3.66 \cdot 10^{-8}$ \\
\hline$\Gamma(100)$ & $W(0.8)$ & 3.270 & 0.367 & 3.580 & $1.33 \cdot 10^{-6}$ \\
\hline$\Gamma(2)$ & $\Gamma(0.49)$ & 2.843 & 0.562 & 2.512 & $4.45 \cdot 10^{-8}$ \\
\hline$\Gamma(2)$ & $\Gamma(1.5)$ & 2.935 & 0.532 & 2.824 & $8.36 \cdot 10^{-5}$ \\
\hline$W(2)$ & $\Gamma(0.49)$ & 2.769 & 0.600 & 2.346 & $4.78 \cdot 10^{-8}$ \\
\hline$W(2)$ & $\Gamma(1.5)$ & 2.880 & 0.562 & 2.706 & $3.27 \cdot 10^{-5}$ \\
\hline
\end{tabular}

is $[0.001,100]$ for the gamma distributions, and it is $[0.001,10]$ for the Weibull distributions. For the distribution $D_{2}$, the maximum interval for the variation coefficient is $[0.001,1.8]$ for the gamma distributions, and it is $[0.001,1.4]$ for the Weibull distributions. 
The results of calculation of reliability steady-state indices for different distributions $D_{1}$ and $D_{2}$ are given in Table 2 . The presented results show that an increase in the variation coefficient of distributions $D_{1}$ and $D_{2}$ leads to an increase in the average number of failed units $N$ and the mean waiting time for recovering $W$ and to a decrease in the stationary availability coefficient of the system $K$.

\section{Conclusions}

This paper shows that the application of hyperexponential approximations of distributions of the units' time to failure and recovery time allow us to calculate steady-state probabilities of the series recoverable system with redundancy and one repair facility. We find these probabilities using solutions of a system of linear algebraic equations obtained by the method of fictitious phases. To obtain parameters of $H_{l}$-approximation of a certain distribution, it is necessary to solve the system of equations of the moments method. Computing the deviations $\Delta_{(6,5)}$ allows us to track the accuracy of approaching distributions $\left\{p_{k(l)}\right\}$ to the true distribution $\left\{p_{k}\right\}$ without the need of using simulation models. We defined conditions for the variation coefficients of the gamma distributions and Weibull distributions, for which the best accuracy of calculating the steady-state probabilities is achieved compared with the case of using simulation models.

\section{References}

1. Aliyev, S. A., Yeleyko, Y. I., Zhernovyi, Yu. V.: Calculating steady-state probabilities of the $G / M / n / m$ queueing systems, Trans. Natl. Acad. Sci. Azerb. Ser. Phys.-Tech. Math. Sci., Mathematics, 39 (4), 9-16 (2019).

2. Aliyev, S. A., Yeleyko, Y. I., Zhernovyi, Yu. V.: Calculating steady-state probabilities of closed queueing systems using hyperexponential approximation, Caspian Journal of Applied Mathematics, Economics and Ecology, 7 (1), 46-55 (2019).

3. Ryzhikov, Yu. I., Ulanov, A. V.: Application of hyperexponential approximation in the problems of calculating non-Markovian queuing systems, Vestnik of Tomsk State Univ. Management, Comp. Eng. and Inform. 36 (3), 60-65 (2016) (in Russian).

4. Ushakov, I.: Probabilistic Reliability Models, John Wiley \& Sons, Hoboken (2012).

5. Zhernovyi, Yu. V., Zhernovyi, K. Yu.: Method of potentials for a closed system with queue length dependent service times, Journal of Communications Technology and Electronics, 60 (12), 1341-1347 (2015).

6. Zhernovyi, Yu. V.: Recurrence relations for two-channel systems with Erlangian service time, Cybernetics and Systems Analysis, 54 (5), 476-483 (2018).

7. Zhernovyi, Yu.: Computing non-Markovian Queues Using Hyperexponential Distributions, LAP Lambert Academic Publishing, Riga (2019).

8. Zhernovyi, Yu.: Creating Models of Queueing Systems Using GPSS World, LAP Lambert Academic Publishing, Saarbrücken (2015).

9. Zhernovyi, Yu. V.: Simulation Models of Reliability, PC "Zhytomyr-Polygraph", Zhytomyr (2020) (in Ukrainian). 